\title{
Abstract: Fiber Optical Shape Sensing of Flexible Instruments
}

\author{
Sonja Jäckle ${ }^{1}$, Tim Eixmann ${ }^{2}$, Hinnerk Schulz-Hildebrandt ${ }^{2,3,4}$, \\ Gereon Hüttmann ${ }^{2,3,4}$, Torben $\mathrm{Pätz}^{5}$ \\ ${ }^{1}$ Fraunhofer MEVIS, Institute for Digital Medicine, Lübeck, Germany \\ ${ }^{2}$ Medizinisches Laserzentrum Lübeck GmbH, Lübeck, Germany \\ ${ }^{3}$ Institute of Biomedical Optics, Universität zu Lübeck, Germany \\ ${ }^{4}$ German Center for Lung Research, DZL, Großhansdorf, Germany \\ ${ }^{5}$ Fraunhofer MEVIS, Institute for Digital Medicine, Bremen, Germany \\ sonja.jaeckle@mevis.fraunhofer.de
}

For minimal invasive procedures like endovascular aortic repair procedures the instruments are navigated with 2D fluoroscopy imaging and digital subtraction angiography, which have several disadvantages. Optical fibers with fiber Bragg gratings (FBG), which allow to sense local strain respectively local curvature and bending angles, can be used for the guidance of medical tools to reduce the X-ray exposure and the used contrast agent. However, FBG-based shape sensing of flexible and long instruments is challenging and the computation includes many steps. In this work, we analyzed in every shape sensing step, which errors can occur, how they affect the resulting shape, and how they can be reduced or corrected [1]. The effects of different methods and parameters were analyzed with experiments done with one multicore fiber of $38 \mathrm{~cm}$ shape sensing length. The results of this analysis were used for an accurate shape sensing model. In an experiment different shapes were measured with the multicore fiber. Then the reconstructed $3 \mathrm{D}$ shapes were compared with the segmented shapes of the acquired CT scans to evaluate the accuracy of our shape sensing model. We obtained an average error of 0.35 to $1.15 \mathrm{~mm}$ and maximal error of 0.75 to $7.53 \mathrm{~mm}$ over the whole $38 \mathrm{~cm}$ sensing length. Furthermore, our shape sensing model was tested in a realistic endovascular setting. Therefore the fiber was inserted into a 3D printed vessel created from patient data. Here, we obtained an average and maximal error of $1.13 \mathrm{~mm}$ and $2.11 \mathrm{~mm}$, respectively. The accuracies of our shape sensing model are promising for using fiber optical shape sensing for catheter guidance. In future work we will use electromagnetic sensors to locate the reconstructed catheter shape.

\section{References}

1. Jäckle S, Eixmann T, Schulz-Hildebrandt H, et al. Fiber optical shape sensing of flexible instruments for endovascular navigation. Int J Comput Assist Radiol Surg. 2019 ;. 